\title{
Laser Micro-Printing of Dye-Molecules on Polymeric Surfaces
}

\author{
Wesley Sims1, Aschalew Kassu ${ }^{2}$, Carlton Farley ${ }^{1}$, Anup Sharma ${ }^{*}$ \\ ${ }^{1}$ Department of Physics, Chemistry and Mathematics, Alabama A\&M University, Normal, AL, USA \\ ${ }^{2}$ Department of Mechanical Engineering, Civil Engineering and Construction Management, \\ Alabama A\&M University, Normal, AL, USA \\ Email: *anup.sharma@aamu.edu
}

Received 24 June 2016; accepted 24 July 2016; published 27 July 2016

Copyright (C) 2016 by authors and Scientific Research Publishing Inc.

This work is licensed under the Creative Commons Attribution International License (CC BY).

http://creativecommons.org/licenses/by/4.0/

(c) $\underset{\mathrm{EY}}{\mathrm{B}}$ Open Access

\section{Abstract}

We describe a technique for micro-patterning and immobilization of dyes on polymer substrates using a low-power visible laser for dye-excitation. Deposits from an aqueous medium containing the dye can be attached at any desired spot on the substrate simply by exposing the area to laser light. The area of the laser beam can control the spot-size of immobilized dye, in the range of 10 100 microns. The immobilization technique is characterized by micro-printing numerals, alphabets and patterns on polybutadiene substrates with Rhodamine (Rh6G) dye. Adsorption of laser-excited dye molecules within the polymer appears to be the mechanism for laser-printing technique.

\section{Keywords}

Laser Micro-Printing, Laser Immobilization of Molecules, Micro-Patterning, Reticle

\section{Introduction}

In recent history, micro-printing is commonly referred to when discussing anti-counterfeiting techniques on items of value. Currencies, IDs and checks are some examples of using very small text, too small for the naked eye, for authenticity. Text or symbols of such small size, usually in the micron range, are extremely difficult to reproduce accurately [1]. Therefore, the use of micro-printing is a feasible technique for anti-counterfeiting an item and confirming its genuineness. Micro-printing proves beneficial in many areas, on many surfaces, for multiple purposes. From serial numbers to bar codes, micro-printing is a ubiquitous necessity in our society. Thus, there have been many techniques reported. Micro-etching and micro-deposition of solid materials, like

\footnotetext{
${ }^{*}$ Corresponding author.
} 
metals and polymers, using ultrashort pulses of ultraviolet (UV) laser radiation have been shown to achieve microstructures of dots, lines and complicated multilevel patterns with submicron resolution [2]. The high-resolution micro-printing techniques have proven to be beneficial for the composition of electronic devices and organic electronic devices [3]. Use of femtosecond excimer lasers produces high definition micron diffractive patterns that can be fabricated on planar and cylindrical substrates such as optical fibers [2].

It has been shown that laser induced forward transfer (LIFT) is suitable for patterning microarrays for proteins as well as antigens without losing any antigenic reactivity or diagnostic properties [4]. In this process, a pulsed laser is used to induce the transfer of material from a laser-transparent source film onto a nearly placed substrate. As the laser pulses propagate through the film and are absorbed by the receptor substrate, material is removed from the source and deposited on the acceptor substrate. Translation of the substrates and/or scanning the laser beam aid in the formation of complex patterns [5]. The LIFT technique is simple and can be used for many solid film materials such as copper and aluminum [5]. The thickness can be adjusted by additional transfers.

In an earlier article, we have reported fabrication of surface relief grating (SRG) patterns from an aqueous phase dye-labeled biomolecule using interferometric lithography using $488 \mathrm{~nm}$ laser light [6] [7]. In this technique, the SRG patterns are formed by immobilizing the molecules in the high intensity region of the spot where the two beams interfere. In another work [8], protein laser printing technique is reported using $100-300 \mathrm{~mW}$ infrared laser light with 1 - 300 seconds of exposure time to activate polymer thin films for micrometer range biopatterning of aqueous phase protein onto laser exposed spots. Step and repeat UV-nanoimprint lithography is also used for nanoscale patterning and fabrication of large area imprinted nanostructured devices by sequentially repeating of the patterning process on spin coated resist films [9]. Matrix assisted pulsed laser evaporation direct write (MAPLE DW) is another technique for micro fabrication of biomolecules, which uses laser light for direct immobilization of biomolecules to a substrate [10]. A non-optical letterpress technique is used for patterning polymer films on curved and flat surfaces. This involves fabrication of micrometer size mechanical and electronic devices by pressing an inked stamp onto the target for applications in the areas where nanometer feature size is not required [11]. More recently, a method for micrometer feature size patterning of polymer surfaces is demonstrated by selective solvent swelling technique [12], whereby the patterning is typically executed by selective and reversible lifting of the polymer surface. Micropatterning of conductive polymer films is done by a method called "printed dissolution" in which polymer films are locally removed followed by stamping and surface processing procedures [13]. The use of inkjet printing [14]-[17] is a widely reported technique for patterning of DNA and protein. Unlike the aforementioned techniques, this article describes a micro-patterning and immobilization technique which involves excitation of dye-molecules in solution with a low-power visible laser, in the vicinity of the polymer substrate. It has the potential to be used with a variety of laser dyes. To the best of our knowledge, this is the first reported micro-printing technique involving embedding of dyes within the polymer surface by using a low power continuous wave visible laser. The technique is primarily presented for its application for printing micro-texts and patterns and for bar-coding although it can also have a potential for immobilizing dye-labeled biomolecules on polymer surfaces for biosensing-related applications.

\section{Materials \& Experimental Section}

The powder form Rhodamine 6 G (Rh6G) purchased from Eastman Kodak Company, Rochester, NY, USA is diluted in distilled water to a concentration of $1 \mathrm{mg} / \mathrm{ml}$. A $2 \%$ by weight solution of polybutadiene rubber in cyclohexane solution, both received from Sigma-Aldrich (St. Louis, MO, U.S.A.), is spin coated at $3000 \mathrm{rpm}$ on microscope glass substrates (Fisher Scientific, Pittsburgh, PA, U.S.A) using spin coating machine from CHEMAT Technology Inc. (Northridge, CA, U.S.A.). The $488 \mathrm{~nm}$ argon-ion laser, with maximum output power of $50 \mathrm{~mW}$ and beam diameter of $0.65 \mathrm{~mm}$, used for the immobilization of Rh6G molecules and micro-printing process was manufactured by Modu-Laser ${ }^{\mathrm{TM}}$ LLC (Centerville, Utah, U.S.A.). To immobilize Rh6G molecules and fabricate patterns over a larger area of polybutadiene coated glass substrates, the laser beam was expanded to $2 \mathrm{~mm}$ diameter using a beam expander (Edmund Optics Inc., Barrington, NJ, U.S.A.). The 21 - $26 \mathrm{~mm}$ in diameter and 1.5 - $2.28 \mathrm{~mm}$ thick contact reticles (Edmund Optics Inc.) with wide range of patterns and sizes were used as masks for microprinting. The patterns on these masks (including alphabets and numerals) have opaque lines printed on transparent glass surface with a line thickness of about $25 \mu \mathrm{m}$.

The schematic experimental setup used for immobilization of dye molecules from aqueous phase and laser micro-printing of pre-patterned shapes using contact-reticles on polybutadiene-coated slides is shown in Figure 1. In Figure 1(a), a small drop of dye-solution is sandwiched between a thin cover-glass substrate $(0.2 \mathrm{~mm}$ 
thickness) and a standard glass slide. The cover-glass as well as the glass-slide is coated with polybutadiene film on the side which is in contact with dye-solution. Hydrophobic nature of the polymer film also keeps the dye-solution trapped between the cover-glass and the glass-slide without flowing away due to capillary action. Thickness of the sandwiched dye-solution is typically $1 \mathrm{~mm}$. The reticle/mask with the pre-etched pattern is loosely placed on top of the cover-glass. An alternative set-up for microprinting by this technique is shown in Figure 1(b). This uses a larger cell filled with the solution of the dye to be immobilized. In this case, the substrate is placed close to the wall, inside the cell with the polymer-coated side facing the incident laser. The reticle/mask is kept adhered outside the cell-wall as shown. While this set-up uses larger amount of dye-solution, it allows printing on multiple substrates without the need for replenishing the solution as is done for set-up 1(a). The optical micrographs of the various metric and multi-gauge contact reticles used for patterning and immobilization of Rh6G molecules on polybutadiene coated substrates are shown in Figure 2.

\section{Results and Discussion}

This section describes the results of visible-light induced micro-printing by embedding a dye within the polymer surface. A summary of micro-printing using numerals, alphabets and other geometrical patterns is given below. Stability of micro-printed patterns under ambient conditions is also described. We use the continuous-wave 488 $\mathrm{nm}$ laser with $40 \mathrm{~mW}$ power for microprinting by this technique. It is observed that laser excitation of Rh6G solution in contact with the polymer substrate results in adsorbed deposit of the dye on the substrate. Thus, as the dye-solution in contact with the substrate is exposed through the reticle, the pattern formed on the polymer film is an exact replica of the transparent regions of the reticle/mask. Depending on the intensity of the writing laser, it takes several minutes for the printed pattern on the polymer to become visible. In general, the rate at which the dye gets implanted in the substrate increases with the intensity of laser light. Results of microprinting on the polymer substrate are shown in Figure 3 for a laser exposure time of 20 minutes. Figures 3(a)-(c) are patterns created using multi-reticle (a), radial lines (b), and numeral "60" (c). In each case, the width of the line is about 25 micron. In Figure 3(d), the increment between divisions is 0.005" (125 micron) and numeral "3" has a width of 250 micron. Figure 3(e) shows a pattern of a metric line with 100 micron increments. The letter "m" extends a width of 3 markings indicating a 300 micron width. Figure 3(f) is pattern created through reticle with double-sided metric lines, in 0.01" increments (top) and in $0.1 \mathrm{~mm}$ increments (bottom). Figure 3(g) shows a

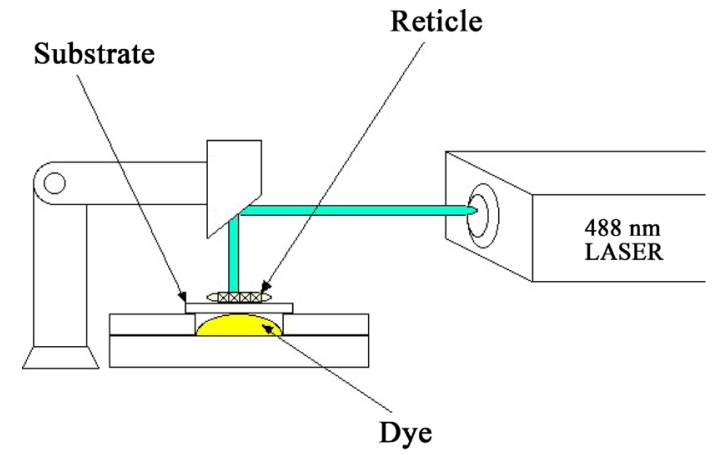

(a)

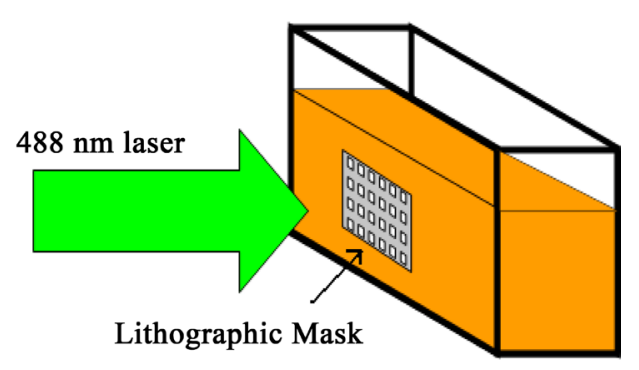

(b)

Figure 1. Schematic experimental set-up for laser micro-printing and immobilization of Rh6G dye and other biomolecules on polymer substrates (a). The alternate set-up using aqueous cell (b) filled with dye-solution.

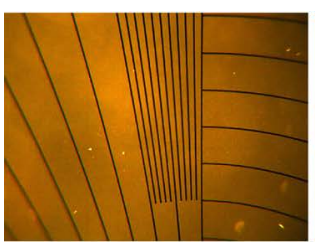

(a)

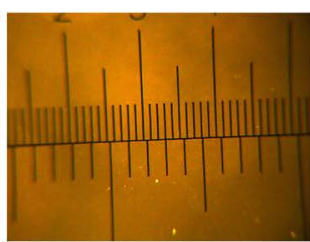

(b)

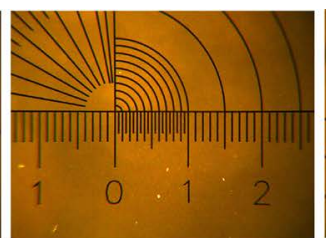

(c)

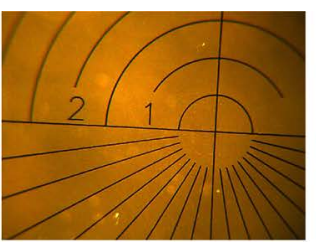

(d)

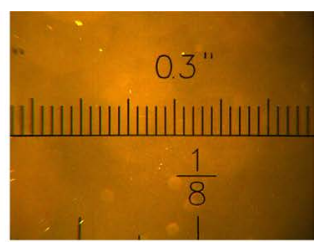

(e)

Figure 2. Optical micrographs of some of the metric and multi-gauge contact reticles used for patterning and immobilization of Rh6G molecules on polybutadiene coated glass slide. 
pattern of radial lines indicating 10 degrees angle in 0.1 degree increments, and Figure $3(\mathbf{h})$ is a pattern of lines with 0.005 " increments. The numeral "2" extends a width of 190 micron.

Soon after laser exposure, the polymer substrate is washed by dipping in water to remove the excess dye-solution sticking to it and then dried before viewing with an optical microscope. The reticles used are designed in such a way that the lines in the patterned surfaces are opaque and the remaining regions are transparent to the $488 \mathrm{~nm}$ laser light. As shown in Figure 3, the patterns are the direct copy of the reticles used, whereby, the dye molecules are immobilized only into the exposed regions of the polybutadiene surface. That is, the lines, letters, figures and dots are the regions where the dye molecules are not attached to the substrates.

It is observed that the micro-printed samples can be stored for several weeks in air without any noticeable degradation. As shown in Figure 4(a), there is no noticeable degradation even if the samples are kept submerged

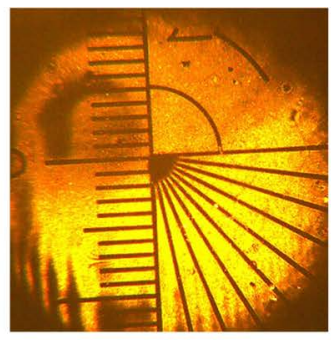

(a)

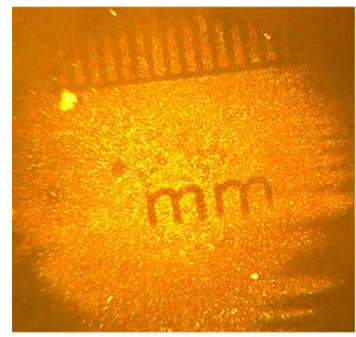

(e)

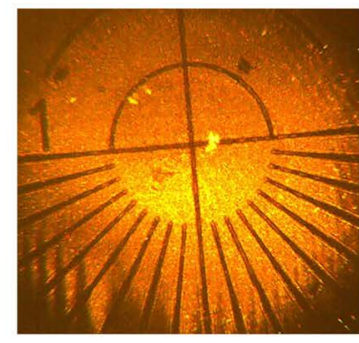

(b)

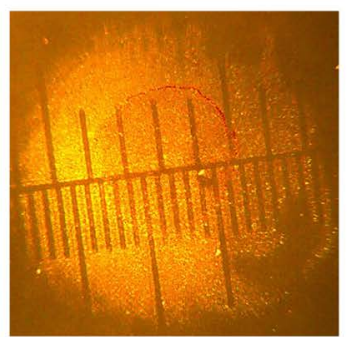

(f)

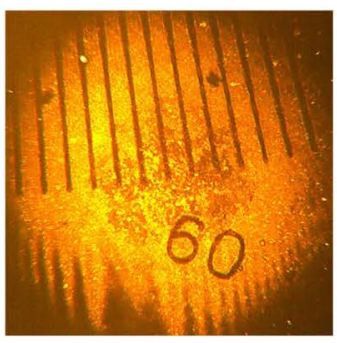

(c)

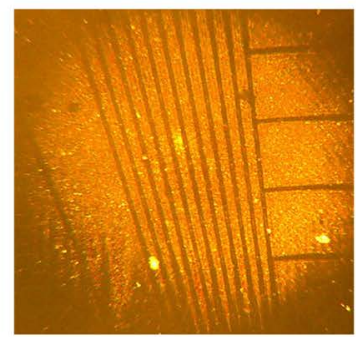

(g)

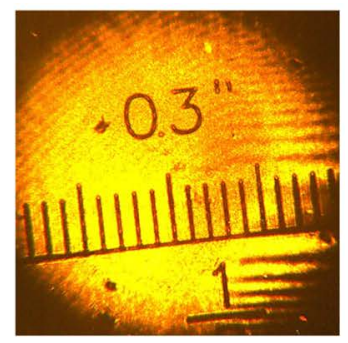

(d)

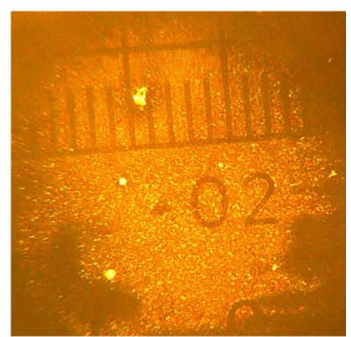

(h)

Figure 3. Illustration of multiple Rh6G patterns created by laser immobilization of Rh6G molecules through multiple reticles into the exposed regions of the PBR surfaces for 20 minutes.

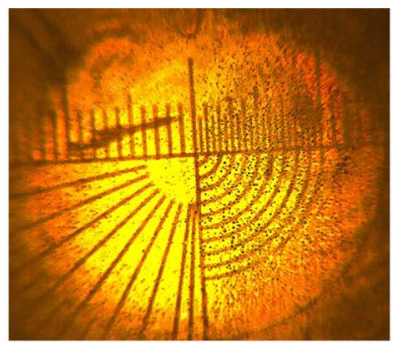

(a)

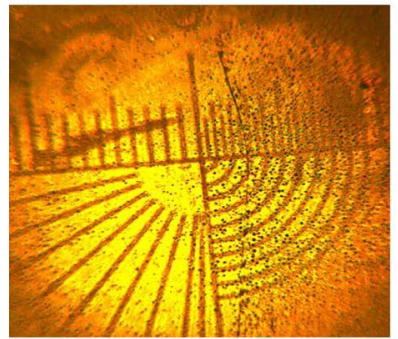

(d)

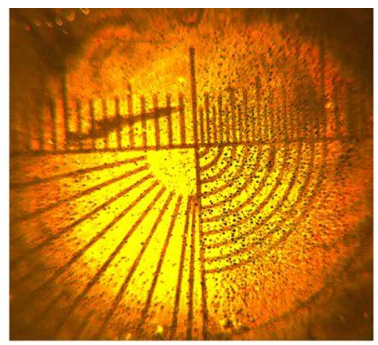

(b)

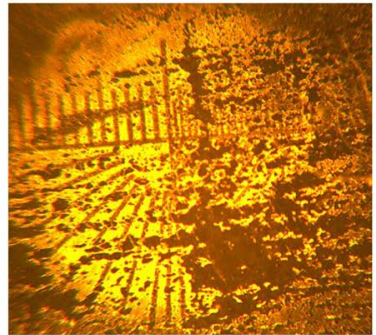

(e)

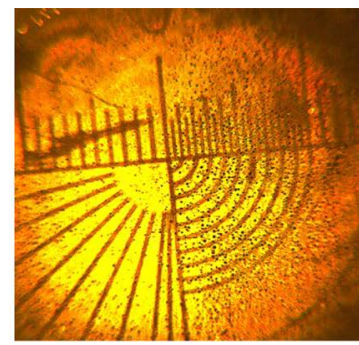

(c)

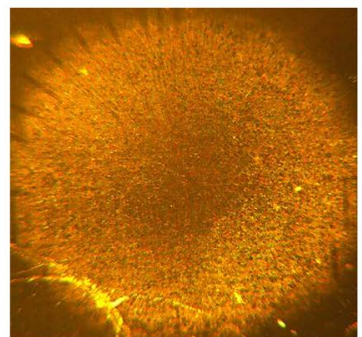

(f)

Figure 4. Effect on dye-immobilized samples stored in water before and after covering the patterns with scotch tape and a spin-coated layer of polybutadiene. 
in water for a few hours. This degradation under water can further be reduced by covering the dye-embedded area with scotch tape (Figure 4(b)) and recoating it with another layer of polymer thin film (Figure 4(c)). Figure 4(d) and Figure 4(e) show the patterns after the spin-coated sample of (c) is immersed in water for 24 hours and for 15 days respectively. Figure 4(f) shows the pattern if it is immersed in water for 24 hours without the second protective coat of polybutadiene thin film. Since any unattached dye-residue can be easily washed away with water in a few seconds, we believe that the dye gets embedded (adsorbed) deeper within the polymer film as a result of dye-excitation with $488 \mathrm{~nm}$ laser light. The technique of micro-printing thus involves enhancement of the adsorption process due to light excitation. However, if kept submerged in water for several hours, the micro-printed pattern fades gradually. This again indicates that the dye is physically adsorbed without forming any strong covalent bond with the polymer.

\section{Conclusion}

In conclusion, a technique for laser micro-printing and immobilization of dye molecules on a polymer film coated substrate is described. The technique is demonstrated with laser excited Rh6G dye and a low-power 488 $\mathrm{nm}$ laser but can work with other laser-dyes and corresponding visible lasers to excite them. We have also shown the technique to work with NBD dye excited with $488 \mathrm{~nm}$ laser although the range of suitable polymer substrates remains to be investigated. When stored in ambient conditions, the patterns formed did not show noticeable degradation for several days. The patterned substrates can also be left in water for a few hours without noticeable degradation and stability under water can be improved by covering it with a scotch tape and recoating with polymer. The technique demonstrated here has a potential for fast, easy and inexpensive micro-printing of lines, dots, letters, symbols, and other complex patterns on polymer substrates and possibly for controlled immobilization and patterning of dye-labeled biomolecules on polymer substrates for biosensing application.

\section{Acknowledgements}

This work was supported by an EPSCoR grant from the National Science Foundation (NSF) [Award Number 1158862].

\section{References}

[1] Trimm, H.H. (2005) Forensics the Easy Way. Barron’s Educational Series, 276.

[2] Zergioti, I., Mailis, S., Vainos, N.A., Ikiades, A., Grigoropoulos, C.P. and Fotakis, C. (1999) Microprinting and Microetching of Diffractive Structures Using Ultrashort Laser Pulses. Applied Surface Science, 138-139, 82-86. http://dx.doi.org/10.1016/S0169-4332(98)00526-1

[3] Bao, Z., Makhjita, A. and Rogers, J.A. (2004) High-Resolution Method for Patterning a Substrate with Micro-Printing. U.S. Patent No. US6736985 B1.

[4] Serra, P., Fernández-Pradas, J.M., Berthet, F.X., Colina, M., Elvira, J. and Morenza, J.L. (2004) Laser Direct Writing of Biomolecule Microarrays. Applied Physics A, 79, 949-952. http://dx.doi.org/10.1007/s00339-004-2577-2

[5] Arnold, C.B., Serra, P. and Piqué, A. (2007) Laser Direct-Write Techniques for Printing of Complex Materials. MRS Bulletin, 32, 23-31. http://dx.doi.org/10.1557/mrs2007.11

[6] Kassu, A., Taguenang, J.M. and Sharma, A. (2008) Photochemically Deposited Surface Relief Gratings of Azo-DyeLabeled Phospholipids from Aqueous Phase. Optics Letters, 33, 1656-1658. http://dx.doi.org/10.1364/OL.33.001656

[7] Sharma, A., Kassu, A., Taguenang, J.M. and Sileshi, S. (2010) Light Activated Immobilization of Dye-Labeled Biomolecules on Polymer Substrates. MRS Spring Meeting, San Francisco, CA, 5-9 April 2010.

[8] Cheng, X., Erdem, E.Y., Takeuchi, S., Fujita, H., Ratner, B.D. and Böhringer, K.F. (2010) Infrared Light Induced Patterning of Proteins on ppNIPAM Thermos-Responsive Thin Films: A "Protein Laser Printer". Lab on a Chip, 10, 1079-1085. http://dx.doi.org/10.1039/b920883f

[9] Peroz, C., Dhuey, S., Volger, M., Wu, Y., Olynick, D. and Cabrini, S. (2010) Step and Repeat UV Nanoimprint Lithography on Pre-Spin Coated Resist Film: A Promising Route for Fabricating Nanodevices. Nanotechnology, 21, No. 44. http://dx.doi.org/10.1088/0957-4484/21/44/445301

[10] Dinu, C.Z., Dinca, V., Howard, J. and Chrisey, D.B. (2007) Printing Technologies for Fabrication of Bioactive and Regular Microarrays of Streptavidin. Applied Surface Science, 253, 8119-8124.

http://dx.doi.org/10.1016/j.apsusc.2007.02.199 
[11] Miller, S.M., Troian, S.M. and Wagner, S. (2002) Direct Printing of Polymer Microstructures on Flat and Spherical Surfaces Using a Letterpress Technique. Journal of Vacuum Science \& Technology B, 20, 2320-2327. http://dx.doi.org/10.1116/1.1520554

[12] Rahmanian, O., Chen, C.F. and DeVoe, D.L. (2012) Microscale Patterning of Thermoplastic Polymer Surfaces by Selective Solvent Swelling. Langmuir, 28, 12923-12929. http://dx.doi.org/10.1021/la302704t

[13] Lind, J.U., Acikgöz, C., Daugaard, A.E., Andresen, T.L., Hvilsted, S., Textor, M. and Larsen, N.B. (2012) Micropatterning of Functional Conductive Polymers with Multiple Surface Chemistries in Register. Langmuir, 28, 6502-6511. http://dx.doi.org/10.1021/la300503p

[14] Rozkiewicz, D.I., Kraan, Y., Werten, M.W.T., de Wolf, F.A., Subramaniam, V., Ravoo, B.J. and Reinhoudt, D.N. (2006) Covalent Microcontact Printing of Proteins for Cell Patterning. Chemistry, 12, 6290-6297. http://dx.doi.org/10.1002/chem.200501554

[15] Voskuhl, J., Brinkmann, J. and Jonkheijm, P. (2014) Advances in Contact Printing Technologies of Carbohydrate, Peptide and Protein Arrays. Current Opinion in Chemical Biology, 18, 1-7. http://dx.doi.org/10.1016/j.cbpa.2013.10.022

[16] Shen, K., Qi, J. and Kam, L.C. (2008) Microcontact Printing of Proteins for Cell Biology. Journal of Visualized Experiments, 22, 1065. http://dx.doi.org/10.3791/1065

[17] Csucs, G., Michel, R., Lussi, J.W., Textor, M. and Danuser, G. (2003) Microcontact Printing of Novel Co-Polymers in Combination with Proteins for Cell-Biological Applications. Biomaterials, 24, 1713-1720. http://dx.doi.org/10.1016/S0142-9612(02)00568-9

\section{Submit or recommend next manuscript to SCIRP and we will provide best service for you:}

Accepting pre-submission inquiries through Email, Facebook, LinkedIn, Twitter, etc.

A wide selection of journals (inclusive of 9 subjects, more than 200 journals)

Providing 24-hour high-quality service

User-friendly online submission system

Fair and swift peer-review system

Efficient typesetting and proofreading procedure

Display of the result of downloads and visits, as well as the number of cited articles

Maximum dissemination of your research work

Submit your manuscript at: http://papersubmission.scirp.org/ 